\title{
Singly-Resonant Optical Parametric Oscillator Based on Orientation-Patterned Gallium Phosphide
}

\author{
Hanyu Ye ${ }^{1}$, S. Chaitanya Kumar ${ }^{2, *}$, Junxiong Wei ${ }^{1}$, P. G. Schunemann ${ }^{3}$, M. Ebrahim-Zadeh ${ }^{1,4}$ \\ ${ }^{1}$ ICFO-Institut de Ciencies Fotoniques, The Barcelona Institute of Science and Technology, 08860 Castelldefels (Barcelona), Spain \\ ${ }^{2}$ Radiantis, Polígon Camí Ral, 08850 Gavà, Barcelona, Spain \\ ${ }^{3}$ BAE Systems, Incorporated, MER15-1813, P.O. Box 868, Nashua, New Hampshire 03061-0868, USA \\ ${ }^{4}$ Institucio Catalana de Recerca i Estudis Avancats (ICREA), Passeig Lluis Companys 23, 08010 Barcelona, Spain \\ *Author e-mail address: chaitanya.suddapalli@ radiantis.com
}

\begin{abstract}
We report a pulsed singly-resonant optical parametric oscillator based on orientationpatterned gallium phosphide pumped by a Q-switched Nd:YAG laser. The mid-IR idler can be tuned across 2.8-3.1 $\mu \mathrm{m}$ with an average power of $20 \mathrm{~mW}$.

OCIS codes: (190.4970) Parametric oscillators and amplifiers; (190.4410) Nonlinear optics, parametric processes; (140.3070) Infrared and far-infrared lasers.
\end{abstract}

Orientation-patterned gallium phosphide (OP-GaP) is a newly emerging semiconductor material for nonlinear frequency conversion into the mid-infrared (mid-IR). Its wide transparency range from as low as $\sim 1 \mu \mathrm{m}$ to $\sim 12 \mu \mathrm{m}$, large nonlinearity $\left(d_{14}=70.6 \mathrm{pm} / \mathrm{V}\right)$, and large bandgap $(2.26 \mathrm{eV}$ at $300 \mathrm{~K})$ allow access to long-wave infrared spectral regions through parametric down-conversion and difference frequency generation in different time-scales using widely available laser pump sources near $\sim 1 \mu \mathrm{m}$. Optical parametric oscillators (OPOs) based on OP-GaP are able to conveniently provide broad wavelength coverage using a single input pump source, but have not been extensively developed to date. In the nanosecond regime, doubly-resonant OPOs based on OP-GaP pumped at $1 \mu \mathrm{m}$ [1] and $2 \mu \mathrm{m}$ [2] have been reported. In the femtosecond time-scale, OP-GaP OPOs pumped at $\sim 1 \mu \mathrm{m}$ [3] and 1.5 $\mu \mathrm{m}$ [4] have also been recently demonstrated. In our work, we report the first singly-resonant pulsed OPO based on OP-GaP pumped by a Q-switched Nd:YAG laser at $1064 \mathrm{~nm}$.

The schematic of the experimental setup for the OP-GaP OPO is shown in Fig. 1(a). The pump source is a linearly polarized, Q-switched Nd:YAG laser at $1064 \mathrm{~nm}$ operating at $50 \mathrm{kHz}$ repetition rate with a pulse duration of $\sim 12$ ns. The pump power can be controlled using an attenuator comprising a half-wave plate and a polarizing beam-splitter (PBS). A second half-wave plate is used for adjusting the pump polarization relative to the [100] direction in the OP-GaP crystal for optimization of output power and efficiency. The OP-GaP crystal, mounted in an oven, is 40 -mm-long, 6-mm-wide, and 1.7-mm-thick, with a single grating period of $\Lambda=16 \mu \mathrm{m}$. The OPO is configured in a compact linear cavity comprising the two plane mirrors, $\mathbf{M}_{1}-\mathrm{M}_{2}$, which are highly reflecting for the signal $(R>99 \%$ over $1300-1900 \mu \mathrm{m}$, and highly transmitting for the pump ( $T>90 \%$ at $1064 \mathrm{~nm})$ and idler $(T>80 \%$ over 2200-4000 $\mu \mathrm{m}$ ), thus ensuring singly-resonant oscillation at the signal wavelength. A long-pass filter with a cut-on wavelength at $2400 \mathrm{~nm}$ is used to extract the generated idler from the transmitted pump. A laboratory photograph of the OP-GaP OPO is shown in Fig. 1(b).
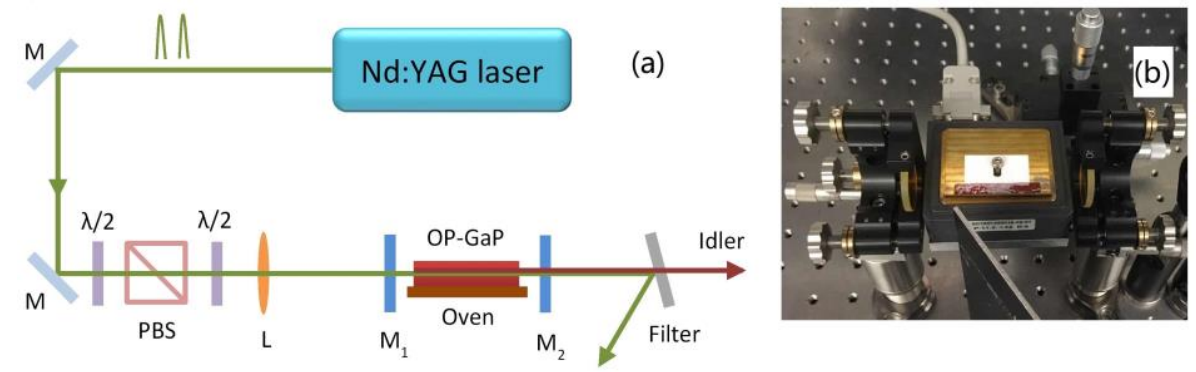

Fig. 1. (a) Schematic of the OP-GaP OPO setup. $\lambda / 2$, half-wave plate; PBS, polarizing beam splitter; $L$, lens. (b) Laboratory photograph of the OP-GaP OPO.

We first investigated the temperature-dependent wavelength tuning characteristics of the OP-GaP OPO. For a pump power of $4.2 \mathrm{~W}$, by increasing the temperature of the OP-GaP crystal from $50{ }^{\circ} \mathrm{C}$ to $180^{\circ} \mathrm{C}$, the $\mathrm{OPO}$ could be tuned across 1612-1700 $\mathrm{nm}$ in the signal and 2845-3130 $\mathrm{nm}$ in the idler, as shown in Fig. 2(a). The signal and idler wavelengths are closely matched with the theoretical calculations based on the Sellmeier equations for OP-GaP given in [1]. We then performed measurements of idler power and pulse energy across the tuning range of the OPO, with the results shown in Fig. 2(b). For an average pump power of $4.5 \mathrm{~W}$, the output idler power and pulse energy 
of the OPO varied from $15.1 \mathrm{~mW}(0.3 \mu \mathrm{J})$ at $2845 \mathrm{~nm}$ to $13 \mathrm{~mW}(0.26 \mu \mathrm{J})$ at $3089 \mathrm{~nm}$, when the crystal temperature was increased from $50{ }^{\circ} \mathrm{C}$ to $160{ }^{\circ} \mathrm{C}$. The highest recorded idler average power was $17.5 \mathrm{~mW}(0.35 \mu \mathrm{J})$ at $2919 \mathrm{~nm}$. The inset of Fig. 2(b) shows the spatial beam profile of the generated idler at $2966 \mathrm{~nm}$, confirming a single-peak intensity distribution.
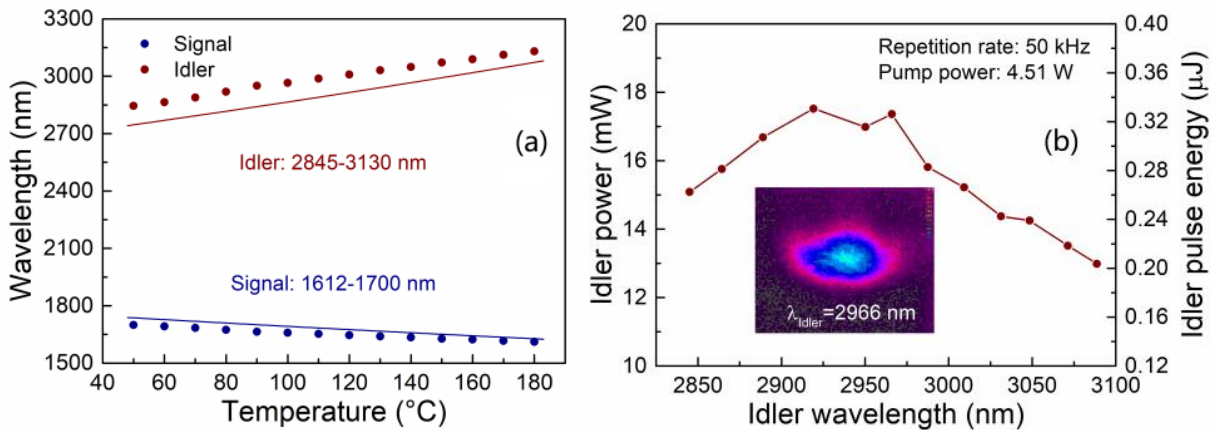

Fig. 2. (a) Wavelength tuning range of the OP-GaP OPO for a grating period of $16 \mu \mathrm{m}$.

(b) Idler power/pulse energy across the wavelength tuning range. Inset: The beam profile of the idler beam.

We also performed idler power scaling measurement at a fixed crystal temperature of $100{ }^{\circ} \mathrm{C}$, with the results shown in Fig. 3 (a). As can be seen, the generated idler power at $2966 \mathrm{~nm}$ increases linearly with a slope efficiency of $1.27 \%$, reaching $19.9 \mathrm{~mW}(0.4 \mu \mathrm{J})$ at a pump power of $4.81 \mathrm{~W}(96.2 \mu \mathrm{J})$. With the OPO in operation, we were able to observe parasitic red light at the exit surface of the OP-GaP crystal. Using a visible spectrometer, we obtained three spectral lines around $648 \mathrm{~nm}, 780 \mathrm{~nm}$ and $833 \mathrm{~nm}$, corresponding to sum-frequency-generation (SFG) between the pump and signal, SFG between the pump and idler, and second harmonic generation (SHG) of the signal, respectively. We further performed power stability measurements of the pump laser and the OPO idler output, with the results shown in Fig. 3(b). The pump laser exhibits a passive power stability of $0.17 \%$ rms with a mean value of $4.81 \mathrm{~W}$ over 1 hour. At a crystal temperature of $100{ }^{\circ} \mathrm{C}$, the corresponding OPO idler output at 2966 $\mathrm{nm}$ exhibits a passive power stability of $0.92 \% \mathrm{rms}$ with a mean value of $20.06 \mathrm{~mW}$ over 1 hour. We also measured the transmission of the pump in the OP-GaP crystal, which was found to be only $\sim 16 \%$.
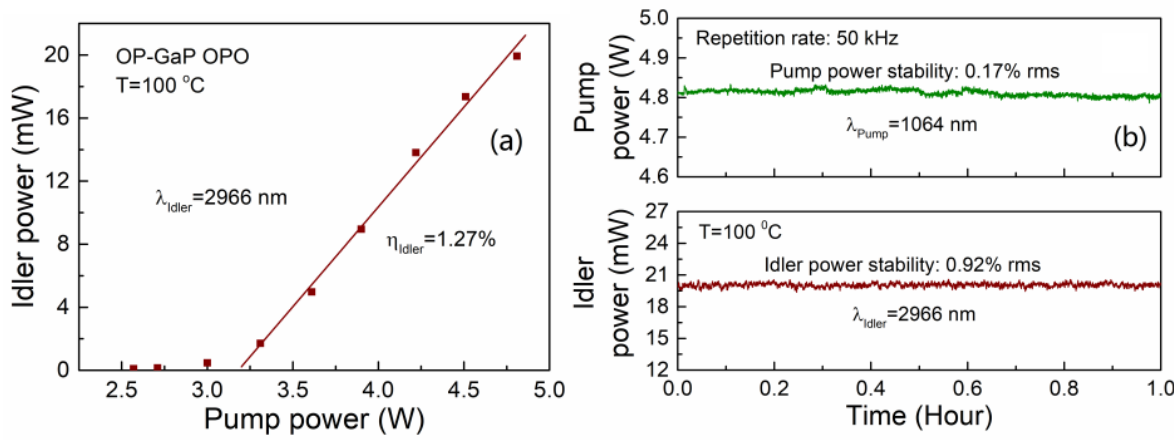

Fig. 3. (a) Idler power scaling as a function of the pump power in the OP-GaP OPO.

(b) Power stability of the pump laser and the idler wave.

In summary, we have demonstrated a pulsed singly-resonant OPO for the mid-IR based on OP-GaP, for the first time, pumped by a Q-switched Nd:YAG laser at $1064 \mathrm{~nm}$ at $50 \mathrm{kHz}$ pulse repetition rate. The OPO provides tunable mid-IR idler output across $2845-3130$ with up to $\sim 20 \mathrm{~mW}$ average power at $2966 \mathrm{~nm}$ and good long-term power stability. With further improvements in the growth and fabrication of the crystal, substantial enhancements in the performance of 1- $\mu \mathrm{m}$-pumped pulsed mid-IR OPOs based on OP-GaP are expected.

\section{References}

[1] L. A. Pomeranz, P. G. Schunemann, D. J. Magarrell, J. C. McCarthy, K. Zawilski, and D. E. Zelmon, "1064-nm-Pumped Mid-Infrared Optical Parametric Oscillator Based on Orientation-Patterned Gallium Phosphide (OP-GaP)," in CLEO: 2015, OSA Technical Digest (online) (Optical Society of America, 2015), paper SW3O.4.

[2] P. G. Schunemann, L. A. Pomeranz, and D. J. Magarrell, "First OPO Based on Orientation-Patterned Gallium Phosphide (OP-GaP)," in CLEO: 2015, OSA Technical Digest (online) (Optical Society of America, 2015), paper SW3O.1.

[3] L. Maidment, P. G. Schunemann, and D. T. Reid, "Molecular fingerprint-region spectroscopy from 5 to $12 \mu \mathrm{m}$ using an orientationpatterned gallium phosphide optical parametric oscillator," Opt. Lett. 41, 4261-4264 (2016).

[4] Q. Ru, Z. E. Loparo, X. Zhang, S. Crystal, S. Vasu, P. G. Schunemann, and K. L. Vodopyanov, "Self-referenced octave-wide subharmonic GaP optical parametric oscillator centered at $3 \mu \mathrm{m}$ and pumped by an Er-fiber laser," Opt. Lett. 42, 4756-4759 (2017). 